have any influence on the rate of penetration of the inhibitors into the cells; the more rapid occurrence of death under the conditions of irradiation must be due to a greater availability of sulphydryl groups. In other words, radiation with wave-lengths greater than $3300 \mathrm{~A}$. causes an increased exposure of intracellular sulphydryl groups.

This conclusion, together with its obvious corollary, of increased cellular susceptibility to attack through its functional systems dependent upon sulphydryl, may offer an explanation for the well-known avoidance of strong light by such free-living organisms as Paramecia. In addition, the above findings are of importance with regard to the mechanism of photodynamic action. This latter point will be discussed in detail elsewhere.

Department of Cancer Research,

G. Calcutt

Mount Vernon Hospital,

Northwood, Middlesex. Apri] 27.

\section{An Exchange between Free Purines and Pyrimidines and the Aglucones of Deoxyribosyl Purines and Deoxyribosyl Pyrimidines}

THE ability of a single deoxyriboside to support growth of several species of bacteria has prompted us to investigate the mechanism of deoxyribosidic interconversion in these organisms. An arbitrary system was adopted which consists of two parts: (1) a crude enzyme preparation from one such organism (Lactobacillus helveticus) ; (2) measurement of the quantity of deoxyriboside (synthesized or destroyed) by microbiological assay with another such organism (Thermobacterium acidophilus $R_{26}$ ). The enzyme preparation contained neither adenine deoxyriboside deaminase nor cytosine deoxyriboside deaminase.

Purine deoxyribosides were differentiated from pyrimidine deoxyribosides by virtue of the resistance of the latter to mild acid-hydrolysis ${ }^{x}$. Individual deoxyribosides were separated by chromatography on paper using $n$-butanol-water-ammonia or $n$ butanol-water-acetic acid systems. Their positions were found under ultra-violet light, and the amounts were determined after cutting out the areas of paper and extracting with water.

By these procedures, it has been possible to demonstrate an exchange of purines and pyrimidines, linked as aglucones to the deoxyribosyl group, with free purines and pyrimidines which are added to the enzyme system. When deoxyribose phosphate ${ }^{2}$ is tested in this system, however, with either purines or pyrimidines, no significant synthesis of deoxyribo. sides takes place. It is therefore concluded that the mechanism of exchange does not involve deoxyribose phosphate as the intermediate.

Thymine, uracil and 5-methyl cytosine (Dr. H. K. Mitchell kindly supplied this compound) have been found to be exchanged with purine deoxyribosides to give pyrimidine deoxyribosides. Chromatography has revealed that the pyrimidine deoxyribosides formed from thymine and uracil have $R_{F}$ values identical with those of thymine deoxyriboside and uracil deoxyriboside respectively. The latter is of interest, since this is the first occasion upon which a biological role has been reported for this compound.
All the usual purines (except uric acid) and 4-amino-5-imidezole carboxamide (prepared from formylmalonamamidine hydrochloride, which was a gift from Dr. D. W. Woolley) react with pyrimidine deoxyribosides to give products which show the acid lability characteristic of purine deoxyribosides. Except for the fact that the product of the reaction between 4-amino-5-imidazole carboxamide and thymine deoxyriboside is not hypoxanthine deoxyriboside, nothing is known of its nature.

Certain other products have also been examined by chromatography on paper. Adenine is exchanged with uracil deoxyriboside to give adenine deoxyriboside. Xanthine is exchanged with uracil deoxyriboside to give a deoxyriboside having an $R_{F}$ value of $0.06-$ a value less than that of any deoxyriboside at present known.

I wish to thaink Dr. E. Hoff-Jørgensen and Dr. H. M. Kalckar for the use of their laboratories, and for their inspiration and help. Mrs. A. L. Ziesler gave technical assistance.

This work was supported by grants from Lederle Laboratories Division, American Cyanamid Co., the Rockefeller Foundation, Carlsbergfondet and the Jane Coffin Childs Memorial Fund for Medical Research, the last-named of which had awarded me a fellowship.

Institutes of Cytophysiology and Watter S. MacNutT Biochemistry,

University of Copenhagen. May 6.

${ }^{1}$ Levene, P. A., and London, E. S., J. Biol. Chem., 83, 799 (1929). 2 Friedkin, M., Kalckar, H. M., and Hoff-Jørgensen, E., J. Biol. Chem.,
178, 527 (1949).

\section{Detection of Sugars on Paper Chromatograms}

THE ammoniacal silver nitrate spray ${ }^{1}$ used for the detection of sugars has several disadvantages; to those mentioned by Partridge ${ }^{2}$ should be added the necessity for very careful enntrol of the heating step, particularly important in laboratories lacking special apparatus. We have introduced modifications, based on a test given by $\mathrm{Feigl}^{3}$ for reducing sugars, which eliminate the heating step, and in which the reagents are applied in organic solvents, thus removing the danger of migration of the sugar spots. The method has been in use for more than a year, and has proved easy to handle and extremely reliable.

In the modified procedure the dried, developed paper chromatogram strip is first passed rapidly through a reagent solution, prepared by diluting $0.1 \mathrm{ml}$. of saturated aqueous silver nitrate solution to $20 \mathrm{ml}$. with acetone, and adding water dropwise, with shaking, until the silver nitrate which separates on addition of acetone has redissolved. Spreading of the spots is limited on account of the sparing solubility of sugars in acetone $\left(0.014\right.$ per cent at $23^{\circ} \mathrm{C}$. for crystalline glucose $\left.e^{4}\right)$. The dry paper is sprayed with a $0.5 \mathrm{~N}$ solution of sodium hydroxide in aqueous ethanol, made by diluting saturated aqueous sodium hydroxide solution with ethanol. Brown silver oxide is immediately produced, the colour facilitating even spraying. 'Reducing-sugars form dense black spots of silver more or less rapidly at room temperature. When reduction is judged complete, excess silver oxide is dissolved by immersing the strip for a few minutes in $6 \mathrm{~N}$ ammonium hydroxide, after which the paper is washed for at least one hour in running 